\title{
ESTUDIO EXPLORATORIO SOBRE LAASOCIACIÓN DE METALES PESADOS Y LA NEFROPATÍA DE ETIOLOGÍA DESCONOCIDA EN EL PONIENTE DEL ESTADO DE MÉXICO
}

\author{
Lilia Patricia BUSTAMANTE-MONTES ${ }^{1}$, Jocsan Alejandro FLORES-POLANCO ${ }^{1}$, \\ Keila ISAAC-OLIVÉ ${ }^{1}$, Marivel HERNÁNDEZ-TÉLLEZ ${ }^{2}$, \\ Martha Elva CAMPUZANO-GONZÁLEZ ${ }^{1}$ y Ninfa RAMÍREZ-DURÁN ${ }^{1 *}$
}

\author{
${ }^{1}$ Facultad de Medicina, Universidad Autónoma del Estado de México, Paseo Tollocan esq. Jesús Carranza s/n, \\ Col. Moderna de la Cruz, 50180 Toluca, Estado de México, México \\ ${ }^{2}$ Centro Interamericano de Recursos del Agua, Facultad de Ingeniería, Universidad Autónoma del Estado de \\ México, Cerro de Coatepec, Ciudad Universitaria, 50110 Toluca, Estado de México, México \\ *Autora para correspondencia: ninfard@hotmail.com
}

(Recibido diciembre 2016; aceptado marzo 2018)

Palabras clave: insuficiencia renal crónica, metales pesados, exposición ocupacional, nefropatía mesoamericana

\begin{abstract}
RESUMEN
En el occidente del Estado de México, la mitad de los ingresos hospitalarios por insuficiencia renal crónica (IRC) no asociada a diabetes mellitus ni a hipertensión arterial corresponden a personas menores de 40 años. Por lo tanto, esta IRC se considera de causas desconocidas (IRCd). En este trabajo se estudió la asociación entre la presencia de metales y factores de riesgo para IRCd. Se realizó un estudio transversal analítico de 100 casos y 100 testigos. Se aplicó un cuestionario para conocer las adicciones y exposiciones (alimentarias, ocupacionales y medicamentosas) de los sujetos. En 50 casos y 50 testigos se determinó la concentración de los metales arsénico, cadmio, mercurio y plomo en muestras de cabello. Finalmente, se construyó un mapa con la posición de la vivienda de los 100 pacientes. Con base en una regresión logística bivariada se determinó que existe una asociación estadísticamente significativa entre la IRCd y el consumo de tabaco, exposición ocupacional a la construcción y herrería, y exposición a arsénico. Es necesaria una muestra mayor para comprobar la exposición ocupacional a la industria del papel, al consumo de paracetamol, naproxeno y cadmio. Los valores obtenidos en este trabajo para estas variables mencionadas son sólo informativos. Los casos estudiados se localizan en la cuenca del Rio Lerma, uno de los más contaminados del país. La IRCd presente en el Estado de México parece ser muy similar a la de Siri Lanka y la del Pacífico centroamericano, por lo que se requieren estudios a mayor escala.
\end{abstract}

Key words: chronic kidney diseases, heavy metals, occupational exposition, Mesoamerican nephropathy

\begin{abstract}
In the western State of Mexico, half of the hospital admissions for chronic kidney diseases (CKD) not associated with diabetes mellitus or hypertension correspond to subjects less than 40 years of age. Therefore, this CKD is considered of unknown causes $(\mathrm{CKDu})$. In this paper we studied whether there was an association between the presence of metals and risk factors with this CKDu. We performed a transversal analytical
\end{abstract}


study of 100 cases and 100 controls. A questionnaire was applied to acknowledge exposition to alcohol, tobacco, drugs, nutrition, or occupational risks. Additionally, we determined in 50 samples and 50 controls the concentration of the metals arsenic, cadmium, mercury and lead. Finally, a map was constructed with the geographic references of housing from 100 patients. After performing a bivariate logistic regression, we determined that there is a statistically significant association between $\mathrm{CKDu}$ and smoking, occupational exposure to construction, blacksmithing, and exposure to arsenic. A larger sample is required to verify the risk of occupational exposure to the paper industry and the consumption of acetaminophen, naproxen, and cadmium. Values obtained in this work for these variables are only informative. The cases studied are in the Río Lerma basin, one of the most contaminated in the country. The CKDu present in the State of Mexico seems to be very similar to that of Siri Lanka and of the Central American Pacific; therefore, larger scale studies are required.

\section{INTRODUCCIÓN}

La enfermedad renal crónica de causas desconocidas (IRCd) es un tipo particular de enfermedad renal crónica (IRC) no relacionada con factores de riesgo como diabetes, hipertensión y síndrome de riñones poliquísticos. Fue inicialmente identificada en Siri Lanka en 1990, y aunque se ha publicado poco al respecto, desde hace aproximadamente dos décadas está afectando de manera severa a países como México (zona suroeste), Guatemala, El Salvador, Honduras, Nicaragua, Costa Rica y Panamá en la costa pacífica de América Central. Por esta razón, la IRCd de Centroamérica es conocida como nefropatía mesoamericana (MeN, por sus siglas en inglés) (Brooks et al. 2012, Jayasumana et al. 2013, Jayatilake et al. 2013, Wesseling et al. 2013, Noble et al. 2014, Redmon et al. 2014, Elinder et al. 2015).

El origen de la MeN y de la IRCd en Sri Lanka aún es desconocido, pero al parecer ambas están relacionadas con factores ocupacionales, tóxicoambientales y hábitos dañinos a la salud renal, combinados con episodios de deshidratación y estrés por calor. Particularmente, el uso de antiinflamatorios no esteroideos (AINE), exposición a arsénico inorgánico, leptospirosis, pesticidas o agua dura son cofactores que influyen en la progresión de esta IRCd, la cual afecta mayoritariamente a hombres y aparece a edades mucho más tempranas que las esperadas para una enfermedad crónica. Es más común en latitudes bajas que altas. En México se ha reportado fundamentalmente en el estado de Chiapas (Brooks et al. 2012, Jayasumana et al. 2013, Jayatilake et al. 2013, Wessling et al. 2013, Noble et al. 2014, Redmon et al. 2014, Elinder et al. 2015).

El Estado de México es la entidad más poblada y densa de México. Su capital, Toluca de Lerdo, ocupa el quinto lugar dentro de las ciudades más pobladas del país; se encuentra ubicada a $2660 \mathrm{msnm}$ y su zona metropolitana también es la quinta mayor del país (Macedo-Miranda et al. 2016). Tiene un clima subtropical de montaña con temperaturas en el rango -3 a $27^{\circ} \mathrm{C}$, con temperatura media anual de $18^{\circ} \mathrm{C}$. Es una zona de alta actividad industrial, caracterizada por la producción de bebidas, comida, textiles, automóviles y productos para la industria eléctrica. El sureste de la región está surcado por el río Lerma (río Lerma-Santiago), el cual es notorio por su contaminación (Zarazúa-Ortega et al. 2013a). En un informe reciente, la Organización Mundial de la Salud (OMS) califica a la ciudad de Toluca como la segunda del país con peor calidad del aire (WHO 2016).

En 2010 se estimaba en México una incidencia de pacientes con IRC de 377 casos por millón de habitantes y una prevalencia de 1142 casos. En ese momento había alrededor de 52000 pacientes en terapia sustitutiva (Méndez-Durán et al. 2010). Las principales causas de IRC en nuestro país son diabetes mellitus (48.5\%), hipertensión arterial (19\%), glomerulopatías crónicas $(12.7 \%)$ y otras $(19.8 \%)$ (Méndez-Durán et al. 2010).

En el Centro Médico Lic. Adolfo López Mateos (CMLALM), hospital de tercer nivel del Instituto de Salud del Estado de México ubicado en Toluca, que atiende a toda la población del poniente del estado, la mitad de los ingresos hospitalarios por IRC corresponden a personas menores de 40 años y no están asociados a diabetes mellitus ni hipertensión arterial. Además, el $10 \%$ de las defunciones en dicha institución se vinculan con IRC en este grupo etario. Esta situación se ha convertido en un problema grave de salud, ya que se presenta en jóvenes aparentemente sanos y en edad económicamente activa.

Una posible causa de esta nefropatía anómala presente en el poniente del Estado de México podría ser la exposición a metales pesados como arsénico, 
cadmio, mercurio y plomo. Otra posible causa es la presencia de IRCd como la MeN, aunque el poniente del Estado de México no cumple con las generalidades descritas anteriormente para este padecimiento, como encontrarse en la costa del Pacífica, altitud baja y clima tropical.

En el centro del país no se han encontrado reportes técnicos ni estudios que relacionen la exposición a metales pesados con la posibilidad de desarrollar IRC. Tampoco hay reportes que indiquen la presencia de IRCd como la MeN. El objetivo de este trabajo fue explorar de manera preliminar si los casos de IRCd no asociada con diabetes mellitus ni hipertensión arterial, presente en pacientes menores de 40 años en el poniente del Estado de México, se vinculan con exposición a metales pesados o si presentan las características comunes a las descritas para la IRCd de Siri Lanka o la MeN de América Central.

\section{MATERIALES Y MÉTODOS}

\section{Muestra}

Estudio transversal analítico pareado por edad ( \pm 5 años). Se incluyeron 100 testigos y 100 casos tratados en el Centro Médico Lic. Adolfo López Mateos del Estado de México, los cuales accedieron a participar firmando una carta de consentimiento informado.

Para conformar el grupo de casos se aplicaron los siguientes criterios de inclusión: pacientes diagnosticados por nefrólogo con insuficiencia renal crónica no asociada a diabetes mellitus ni hipertensión arterial (IRCd), y edad de 15 a 40 años de ambos sexos. Para los testigos se realizó un muestreo no probabilístico a conveniencia, seleccionando a familiares que acompañaban a los pacientes a consulta, clínicamente sanos, quienes también firmaron la carta de consentimiento informado.

Para el grupo de casos se excluyeron todos aquellos pacientes que padecían patologías previas que favorecieran el desarrollo de insuficiencia renal crónica, pacientes que no desearan seguir participando en el estudio durante el desarrollo de éste, y casos incidentes que no contaron con un control. En cuanto al grupo de testigos, se excluyó a familiares con cualquier tipo de patología o que no firmaran el consentimiento informado. Estudio de la exposición a adicciones, alimentaria,
ocupacional y medicamentosa

Tanto a los 100 pacientes como a los 100 casos se les aplicó un cuestionario enfocado a conocer adicciones, exposición a alimentos, exposición ocupacional y exposición a medicamentos nefrotóxicos. La base de datos construida se analizó utilizando el paquete estadístico Stata v. 11.0. Se realizó un análisis exploratorio y limpieza de los datos. Se procedió al análisis descriptivo de cada variable. Se realizó una regresión logística bivariada no condicional entre cada una de las variables independientes y la variable dependiente. Se seleccionaron como variables estadísticamente significativas todas aquellas con valor de $\mathrm{p}<0.05$. Se determinó para cada variable la razón de momios (RM). En este estudio la variable dependiente es la presencia o ausencia de IRCd, y las independientes son las siguientes: consumo de tabaco, alcohol, pescado o atún; exposición laboral a materiales de construcción, herrería, industria del papel, floricultura y gasolineras, y exposición medicamentosa a paracetamol y naproxeno (AINE).

\section{Estudio de la exposición a metales pesados nefro- tóxicos: arsénico, cadmio, mercurio y plomo}

A todos los pacientes y testigos se les tomó una muestra de cabello. Los reactivos utilizados para obtener dicha muestra fueron: acetona $99.5 \%$ de grado analítico y agua para análisis de buena calidad. El instrumental de corte, como las tijeras y el bisturí, fueron de grado quirúrgico, y se utilizó un área de trabajo limpia y libre de fuentes de contaminación.

La muestra de cabello se obtuvo de la región más recomendada del cuero cabelludo, que es la occipital $\mathrm{y}$, dentro de ella, la vértex posterior. Antes de proceder a la toma de muestra, se limpiaron las tijeras con acetona para eliminar todo vestigio de grasa y polvo en las superficies de corte. El personal que tomó la muestra realizó un lavado de manos antes de proceder a la recolección.

Se tomaron de cinco a 10 hebras de pelo en 20 lugares diferentes de la región occipital homogéneamente distribuidos. De esta forma, al final del muestreo se contaba con al menos 100 hebras de pelo. El corte se realizó lo más cerca posible del cuero cabelludo. Una vez recolectadas, todas las hebras se colocaron sobre una lámina de vidrio y se alinearon respetando la dirección de las mismas. A partir de la punta más cercana al cuero cabelludo se realizó un corte a los $5 \mathrm{~cm}$ con un bisturí. Esta porción de cabello fue lavada con acetona y agua en tres ciclos diferentes. Luego se secó en una estufa a $70{ }^{\circ} \mathrm{C}$.

De las 100 muestras pertenecientes a casos y las 100 muestras testigos se escogieron aleatoriamente 50 casos y 50 testigos. Estas 100 muestras se enviaron al Laboratorio Nacional de Biotecnología Agrícola 
Médica y Ambiental del IPICyT (Instituto Potosino de Investigación Científica y Tecnológica) para realizar la determinación correspondiente de metales pesados (arsénico, cadmio, mercurio y plomo) mediante la técnica de plasma acoplado inductivamente con detección mediante espectroscopia de emisión atómica (ICP-AES).

De manera similar al caso anterior, la variable dependiente es la IRCd y las independientes la exposición a cada metal estudiado. Se seleccionaron como variables estadísticamente significativas todas aquellas con un valor de $\mathrm{p}<0.05$. Se determinó para cada variable la razón de momios (RM) y su intervalo de confianza al $95 \%$ de nivel de significancia.

\section{Distribución geográfica de la enfermedad}

Las coordenadas de las viviendas de los $100 \mathrm{pa}-$ cientes diagnosticados con IRCd se capturaron con el software Idrisi Selva. Las imágenes del satélite se obtuvieron de Glovis (http://glovis.usgs.gov/), y se seleccionó una imagen Landsat como imagen de fondo. Sobre ésta se señalaron las coordenadas de todos los casos obtenidas del programa Google Earth y se agregó la cuenca del río Lerma y los cuerpos de agua más representativos del Estado de México, ocupando los límites poblacionales establecidos por el Instituto Nacional de Estadística y Geografía (INEGI). El sistema de referencia utilizado es UTM zona 14 con datum WGS84.

\section{RESULTADOS}

Relación de la IRC con exposición alimentaria, ocupacional y medicamentosa

El cuadro I muestra la relación entre IRCd y exposición a adicciones, consumo de pescado, exposición ocupacional y uso de medicamentos nefrotóxicos. En el mismo se muestran las razones de momios y su intervalo de confianza al $95 \%$. Asimismo, en el cuadro I se observa que las variables estadísticamente significativas, cuyo intervalo de confianza de la razón de momios calculada no incluye el cero, son: consumo de tabaco $(\mathrm{p}=0.001)$; productos del trabajo relacionados con la construcción $(\mathrm{p}=0.03)$, y herrería $(p=0.02)$. Por lo tanto, estas variables influyen significativamente en el desarrollo de la IRCd y el riesgo correspondiente a cada una es de 2.74, 2.70 y 10.89 , respectivamente. Este resultado evidencia que la ocupación laboral es un factor importante en el desarrollo de la enfermedad.

La industria del papel tuvo una razón de momios mayor a $2, p=0.05$ y un intervalo de confianza muy amplio en el que el límite inferior es muy cercano a 1. Algo similar ocurre con el consumo de medicamentos y la ocupación laboral en gasolineras. En el primero, las probabilidades obtenidas son de 0.06 , muy cercanas al valor crítico de 0.05 , y en la ocupación laboral la probabilidad obtenida no es cercana a 0.05 pero el intervalo de confianza obtenido es muy amplio. Estos

CUADRO I. ANÁLISIS DE LA RELACIÓN ENTRE ADICCIONES, ALIMENTOS, EXPOSICIÓN OCUPACIONAL Y MEDICAMENTOS E INSUFICIENCIA RENAL CRÓNICA

\begin{tabular}{|c|c|c|c|c|c|c|c|c|}
\hline \multirow{2}{*}{ Factor } & \multirow{2}{*}{ Variable } & \multicolumn{2}{|c|}{ Pacientes con IRCd } & \multicolumn{2}{|c|}{ Grupo control } & \multirow{2}{*}{$\mathrm{RM}$} & \multirow{2}{*}{$\mathrm{p}$} & \multirow{2}{*}{ IC de RM } \\
\hline & & $\mathrm{E}$ & $\mathrm{NE}$ & $\mathrm{E}$ & $\mathrm{NE}$ & & & \\
\hline \multirow{2}{*}{ Adicciones } & Tabaco & 45 & 55 & 23 & 77 & 2.74 & 0.001 & $(1.48,5.04)$ \\
\hline & Alcohol & 13 & 69 & 8 & 78 & 1.84 & 0.19 & $(0.66,5.42)$ \\
\hline \multirow[t]{2}{*}{ Alimentos } & Pescado & 12 & 88 & 11 & 89 & 1.10 & 0.39 & $(0.86,1.43)$ \\
\hline & Atún & 83 & 17 & 84 & 16 & 0.93 & 0.83 & $(0.60,1.48)$ \\
\hline \multirow{5}{*}{$\begin{array}{l}\text { Exposición } \\
\text { ocupacional }\end{array}$} & Construcción & 19 & 81 & 8 & 92 & 2.70 & 0.03 & $(1.07,5.20)$ \\
\hline & Herrería & 9 & 81 & 1 & 98 & 10.89 & 0.02 & $(1.34,92.57)$ \\
\hline & Papel & 54 & 44 & 20 & 79 & 4.85 & 0.05 & $(0.96,24.66)$ \\
\hline & Floricultura & 83 & 17 & 84 & 16 & 0.93 & 0.93 & $(0.14,5.80)$ \\
\hline & Gasolineras & 5 & 91 & 1 & 98 & 5.38 & 0.13 & $(0.60,51.70)$ \\
\hline \multirow[t]{2}{*}{ Medicamentos } & Paracetamol & 35 & 61 & 20 & 79 & 2.27 & 0.06 & $(0.96,5.19)$ \\
\hline & Naproxeno & 7 & 93 & 2 & 98 & 3.69 & 0.06 & $(0.94,12.23)$ \\
\hline
\end{tabular}

IRCd: insuficiencia renal crónica de causa desconocida; RM: razón de momios; p: probabilidad de desarrollar IRCd al estar expuesto al factor en estudio; IC: intervalo de confianza; E: expuestos; NE: no expuestos 
CUADRO II. RANGO DE CONCENTRACIONES Y FRECUENCIA DE APARICIÓN DE LOS METALES PESADOS ARSÉNICO, CADMIO, PLOMO Y MERCURIO EN CASOS Y TESTIGOS

\begin{tabular}{ccccc}
\hline $\begin{array}{c}\text { Metal y su límite } \\
\text { de detección } \\
(\mathrm{ng} / \mathrm{g})\end{array}$ & $\begin{array}{c}\text { Frecuencia de } \\
\text { detección del metal } \\
\text { en 50 pacientes }\end{array}$ & $\begin{array}{c}\text { Promedio y rango de } \\
\text { concentraciones en } \\
\text { pacientes }(\mu \mathrm{g} / \mathrm{g})\end{array}$ & $\begin{array}{c}\text { Frecuencia de } \\
\text { detección del metal } \\
\text { en } 50 \text { testigos }\end{array}$ & $\begin{array}{c}\text { Promedio y rango de } \\
\text { concentración en testigos } \\
(\mu \mathrm{g} / \mathrm{g})\end{array}$ \\
\hline As: 42 & $50(11)^{\mathrm{a}}$ & $0.59(0.07-1.76)$ & $50(3)^{\mathrm{a}}$ & $0.41(0.07-1.60)$ \\
$\mathrm{Cd}: 25$ & 48 & $0.13(0.00-0.88)$ & 50 & $0.09(0.00-0.27)$ \\
$\mathrm{Pb}: 23$ & 48 & $13.31(1.52-53.40)$ & 50 & $12.58(2.94-25.63)$ \\
Hg: 3 & 1 & $0.27(0.00-0.27)$ & 11 & $0.31(0.00-1.46)$ \\
\hline
\end{tabular}

Nota: Las concentraciones promedio reportadas de los metales en pacientes y testigos se calcularon con base en la frecuencia de detección encontrada en cada grupo para cada metal

${ }^{a}$ Número de pacientes o testigos con concentración mayor a $1 \mu \mathrm{g} / \mathrm{g}$, valor máximo permitido de As en cabello humano según la OMS (Carvalho et al. 2009)

valores son indicativos de que dichas actividades podrían ser factores de riesgo, pero el tamaño de la muestra no fue lo suficientemente grande. Así que se recomienda ampliar el tamaño de la misma.

\section{Relación de la IRCd con exposición a metales pesados nefrotóxicos: arsénico, cadmio, mercurio y plomo}

El cuadro II muestra los resultados del promedio y rango de las concentraciones de los metales encontradas en el cabello de los casos y los testigos, así como la frecuencia de aparición de dichos metales en ambos grupos. Más del $96 \%$ de los casos y testigos estudiados están expuestos a arsénico, cadmio y plomo, y en estos metales los casos tienen concentraciones promedio ligeramente mayores y rangos más amplios que el grupo control. El caso del mercurio resultó anómalo con relación a los otros metales, ya que si bien su presencia fue en general baja, tanto su frecuencia como su concentración fueron más altas en testigos que en pacientes.

La concentración de arsénico en cabello reportada en este trabajo es superior a la encontrada en otras poblaciones, como Altamira (Brasil, $0.434 \mu \mathrm{g} / \mathrm{g}$ ), Canadá y Estados Unidos $(0.001 \mu \mathrm{g} / \mathrm{g})$, Polonia $(0.020 \mu \mathrm{g} / \mathrm{g})$, Italia $(0.090 \mu \mathrm{g} / \mathrm{g})$, Portugal y Nigeria $(0.040 \mu \mathrm{g} / \mathrm{g})$, Japón $(0.23 \mu \mathrm{g} / \mathrm{g})$ y Malasia $(0.28$ $\mu \mathrm{g} / \mathrm{g})$. Sólo fue menor que la reportada en la India $(0.610 \mu \mathrm{g} / \mathrm{g})$ (Carvalho et al. 2009). Al compararla con la de pacientes con IRCd en Sri Lanka y un grupo control de una zona endémica de IRCd en ese país, se obtuvieron los siguientes datos: pacientes con IRCd de Sri Lanka: $\mathrm{n}=80$, media $0.144 \mu \mathrm{g} / \mathrm{g}$, mediana $0.001 \mu \mathrm{g} / \mathrm{g}$, rango $0.00-0.452 \mu \mathrm{g} / \mathrm{g}$; grupo control de zona endémica: $\mathrm{n}=48$, media $0.125 \mu \mathrm{g} / \mathrm{g}$, mediana $0.103 \mu \mathrm{g} / \mathrm{g}$, rango $0.006-1.214 \mu \mathrm{g} / \mathrm{g}$. Como se puede observar en el cuadro II, el valor de la media encontrada en pacientes y testigos del poniente del
Estado de México es aproximadamente cuatro veces mayor que la reportada en Sri Lanka para pacientes con IRCd (Jayatilake et al. 2013).

Sin embargo, el cadmio se comportó de forma diferente. La concentración promedio de este metal encontrada en el cabello de pacientes y testigos en este estudio fue inferior a la reportada en el cabello de otras poblaciones (Carvalho et al. 2009). En el caso del plomo, en Isla Canteneiro (Brasil), India e Indonesia se encontraron valores de 23.2, 13.2 y 15.7 $\mu \mathrm{g} / \mathrm{g}$, respectivamente, superiores a los encontrados en el poniente del Estado de México. El resto de los sitios mencionados con anterioridad reportaron valores inferiores a los de nuestro estudio. Este resultado sugiere que debe establecerse el nivel de plomo en sangre para determinar si hay toxicidad debida a este metal en la región de estudio. La concentración promedio de mercurio encontrada en este trabajo fue siempre inferior a la reportada en otras poblaciones estudiadas (Carvalho et al. 2009).

El cuadro III muestra los resultados del análisis de exposición a metales asociada con IRCd en el poniente del Estado de México, realizado en el cabello de 50 casos y 50 testigos seleccionados al azar entre

CUADRO III. ANÁLISIS DE LA EXPOSICIÓN A LOS METALES PESADOS ARSÉNICO, CADMIO, PLOMO Y MERCURIO CON INSUFICIENCIA RENAL CRÓNICA

\begin{tabular}{lrcc}
\hline Metal & RM & $p$ & IC de RM \\
\hline Arsénico & 3.20 & 0.04 & $(1.02,9.98)$ \\
Cadmio & 37.21 & 0.15 & $(0.26,5322)$ \\
Plomo & 1.02 & 0.48 & $(0.96,1.09)$ \\
Mercurio & 0.34 & 0.72 & $(0.001,1445)$ \\
\hline
\end{tabular}

RM: razón de momios, p: probabilidad de desarrollar IRCd al estar expuesto al metal en estudio, IC: Intervalo de confianza 
los 100 casos y 100 testigos iniciales.

Aun cuando la diferencia entre los rangos de concentraciones encontrados en pacientes y testigos para el arsénico no es amplia (cuadro III), fue suficiente para determinar que la exposición a este metal es significativa, ya que el riesgo de desarrollar IRCd es tres veces superior en presencia de exposición a arsénico. De acuerdo con la OMS, el límite máximo permitido de este elemento en cabello humano es de $1 \mu \mathrm{g} / \mathrm{g}$ (Carvalho et al. 2009). En el cuadro II se indica que 11 pacientes y tres testigos presentaron valores mayores al permitido por la OMS en las muestras de cabello, lo que se traduce a que $22 \%$ de la población con IRCd presenta concentraciones tóxicas de arsénico. Estos 11 pacientes están distribuidos de la siguiente manera: dos residen y trabajan en el municipio de Almoloya de Juárez, tres residen y trabajan en el municipio de Toluca, uno reside y trabaja en el municipio de Zinacantepec, uno reside y trabaja en el municipio de Temoaya, uno reside en Toluca y trabaja en Ixtlahuaca, uno reside en Villa Cuauthémoc y trabaja en Otzoloapan, y finalmente dos residen en Jiquipilco y trabajan en Toluca.

El cadmio es un elemento conocido por su nefrotoxicidad. Las concentraciones de este metal encontradas en el presente estudio y reportados en el cuadro II, son del mismo orden que las encontradas trabajos similares (Carvalho et al. 2009). Las concentraciones promedio de ambos grupos no están muy distantes; sin embargo, la razón de momios fue de 37 , lo cual indica que la exposición a este metal aumenta 37 veces la posibilidad de contraer IRCd. El valor máximo permitido de este metal en cabello humano no se ha establecido, por lo que no es posible conocer el porcentaje de pacientes con IRCd que presentan toxicidad por este elemento.

Pese al alto valor de la fracción de momios, ésta no fue estadísticamente significativa con un nivel de confianza de $95 \%$, pero el intervalo de confianza fue muy amplio (0.26 a 5322), lo cual indica que este valor de riesgo debe tomarse como informativo y el estudio debe repetirse con una muestra más amplia. Vale la pena indicar que de los 10 casos con concentraciones mayores de cadmio ninguno fuma, por lo que la principal exposición a este metal no es vía tabaco. De los 11 pacientes que presentan intoxicación por arsénico, seis pertenecen al cuartil de concentraciones más altas de cadmio, y residen o laboran en los municipios de Toluca, Zinacantepec, Almoloya de Juárez y Jiquipilco.

Aunque el plomo no está asociado a IRCd en Sri Lanka o MeN en América Central, las concentra- ciones encontradas de este metal tanto en pacientes como en testigos son relativamente altas en comparación con las informadas en otras partes del mundo (Carvalho et al. 2009). Sin embargo, no lo suficiente para asegurar la presencia de toxicidad debida a este metal, ya que los valores mínimos de este metal se han establecido para sangre, no para cabello. Las concentraciones y rangos en pacientes y testigos son similares, exceptuando un paciente que alcanzó un valor de $53.4 \mu \mathrm{g} / \mathrm{g}$ y amplió el rango en su el grupo de pacientes. Excluyendo este valor, ambos rangos son semejantes. Estas similitudes entre grupos impiden detectar diferencias, razón por la cual la razón de momios para el plomo es unitaria y el valor de la probabilidad está muy lejos de considerarse estadísticamente significativo.

Es importante aclarar que varios estudios recientes indican la presencia de plomo en el aire de la zona metropolitana del valle de Toluca, lo cual justifica el alto contenido de este metal encontrado en cabello de pacientes y testigos (Zarazúa-Ortega et al. 2013b, Macedo-Miranda et al. 2016). El paciente que presentó el valor extremo de plomo (53.4 $\mu \mathrm{g} / \mathrm{g}$ ) reside y labora en Toluca y su ocupación es la construcción.

Los resultados obtenidos a partir del mercurio sólo permiten concluir que, de los cuatro metales analizados, es al que menos expuesta está la población estudiada. Cualquier otro resultado es meramente informativo, dado que el tamaño de muestra resultó insuficiente para llegar a otras conclusiones. La exposición humana a los compuestos de mercurio está ligada exclusivamente al consumo de pescado y productos derivados. Por sus condiciones geográficas y costumbres culturales, el Estado de México no tiene un alto consumo de estos productos, por lo que se requiere un tamaño de muestra mucho mayor. El único paciente al que se le encontró mercurio en la muestra de cabello reside y estudia en Toluca. En el caso de los 11 testigos que presentaron mercurio en su cabello, sus lugares de residencia son: Toluca (3), Otzoloteoec (2), Temoaya (1), Tenango (1), Almoloya de Juarez (1), Jiquipilco (1), Metepec (1) y Atlacomulco (1). El valor más alto encontrado fue de $1.46 \mu \mathrm{g} / \mathrm{g}$.

\section{Diseño del mapa de distribución geográfica de la enfermedad}

La figura 1 muestra la distribución de los pacientes con diagnóstico de IRCd. La mayor concentración de sujetos se localiza en los municipios de Toluca, Almoloya de Juárez, Ixtlahuaca, Zinacantepec, Metepec, Xiquipilco, Otzolotepec, Lerma y Atlacomulco. La distribución de los pacientes 


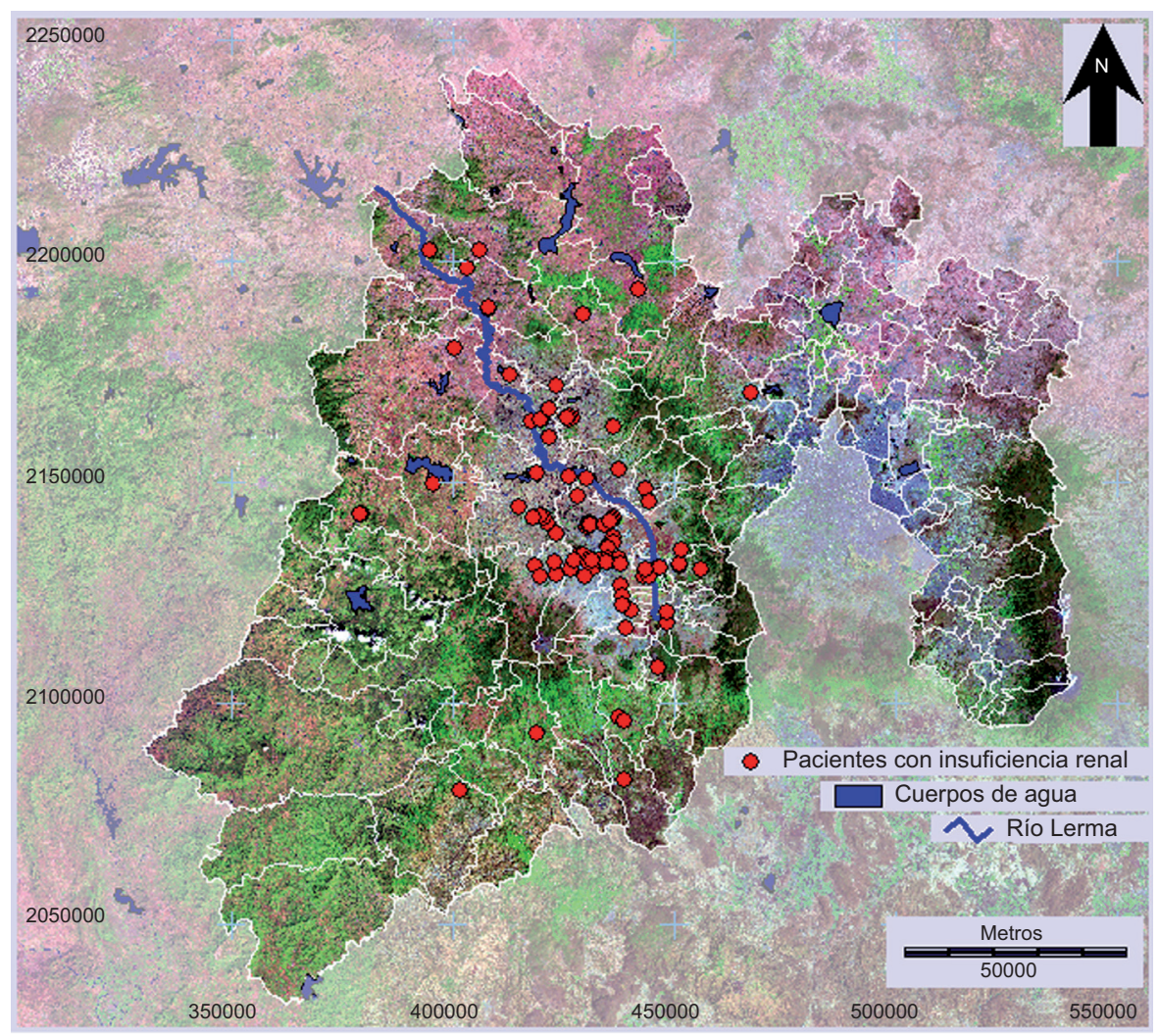

Fig. 1. Mapa de distribución de pacientes con insuficiencia renal crónica atendidos en el Centro Médico Lic. Adolfo López Mateos ubicado en Toluca de Lerdo, Estado de México

coincide con la cuenca del rio Lerma, uno de los ríos más contaminados de la región por metales pesados (Zarazúa et al. 2013a).

Varios estudios reportan la determinación de metales pesados tanto en agua como en sedimento en diferentes segmentos del río Lerma. Barceló-Quintal et. al. (2012) determinaron la concentración de plomo en aguas en varias zonas del río. Se encontraron concentraciones en el rango de $25-60 \mathrm{ng} / \mathrm{mL}$, excepto en el cruce del río con la carretera México-Toluca a la altura de San Mateo Atenco, donde la concentración encontrada fue de $225 \mathrm{ng} / \mathrm{mL}$. En sedimentos se han reportado las siguientes concentraciones: plomo, 12-64 ng/g (Tejeda et al. 2006); cadmio, $9.6 \mathrm{ng} / \mathrm{g}$ (Brito et. al. 2015); mercurio, 0.07-1.18 $\mu \mathrm{g} / \mathrm{g}$ (Trasande et. al. 2010); y arsénico, 0.5-4 $\mu \mathrm{g} / \mathrm{g}$ (Aburto-Medina et al. 2015). Para el caso del plomo y el cadmio, estos valores son mucho menores que los encontrados en cabellos de los pacientes. Sin embargo, en el caso del mercurio y el arsénico son del mismo orden, teniendo en cuenta que concentraciones de arsénico mayores a $1 \mu \mathrm{g} / \mathrm{g}$ en cabello son consideradas tóxicas (Carvalho et al. 2009).

\section{DISCUSIÓN}

Los resultados de este trabajo indican que la IRCd en pacientes de 15 a 40 años de edad, sin antecedentes de diabetes mellitus ni hipertensión arterial, residentes en el poniente del Estado de México (se incluye la zona metropolitana del Valle de Toluca), está asociada a factores como consumo de tabaco, exposición laboral (construcción, herrería y en general trabajo pesado), así como exposición al metal nefrotóxico arsénico. Por el tamaño de la muestra, se reporta como informativo el consumo de antiinflamatorios (AINES), el trabajo en la industria del papel y gasolineras, y la exposición a cadmio. Estos resultados coinciden con la descripción de la IRCd en Sri Lanka y la MeN en Centroamérica; por lo mismo, sugieren que una variante de la IRCd o MeN está presente en el poniente del Estado de México.

La MeN está descrita en regiones costeras del Pacífico tropical, y se le asocia con estrés por deshidratación y trabajo pesado a altas temperaturas. Estas condiciones no se dan en el poniente del Estado de 
México. Toluca de Lerdo, su capital, está lejos de la costa y su clima es subtropical. Sin embargo, en el presente estudio no se investigaron las temperaturas en el lugar de trabajo de los pacientes y, dado que la exposición ocupacional se encontró fundamentalmente en herreros, constructores y obreros de la industria del papel, es probable que dichas temperaturas sean elevadas. En el caso de Sri Lanka, la IRCd se ha encontrado en regiones interiores de la isla y no siempre a lo largo de la costa.

Es sabido que los AINE, muy utilizados en la actualidad en el tratamiento de hipertensión e insuficiencia cardíaca, así como en la prevención secundaria de infarto de miocardio, producen con cierta frecuencia IRC (Plantinga et al. 2011). Este estudio no incluyó a pacientes de IRC con antecedentes de hipertensión arterial o insuficiencia cardíaca; por lo tanto, no estaban expuestos a los AINE por esta razón. Sin embargo, este tipo de medicamentos se utilizan comúnmente para tratar dolor muscular (común en personas vinculadas con trabajo pesado), síntomas de gripe, dolor de cabeza, etc. Los pacientes en este estudio si refirieron consumirlos con cierta frecuencia.

La población estudiada está expuesta a arsénico, pero en principio no al cadmio. Este último no se encontró en muestras de cabello en concentraciones mayores que las reportadas en cabello en otras partes del mundo. Sin embargo, aunque no fue significativa, su razón de momios resultó ser muy alta, lo cual supone la presencia del fenómeno ya descrito de coexposición de arsénico y cadmio, en que el efecto nefrotóxico de la exposición aditiva a ambos metales es mayor que la exposición a cada metal de forma individual (Jayatilake et al. 2013) Se ha reportado que la exposición ambiental baja a cadmio no es significativa en el caso de la IRCd y la MeN, pero sí en el caso de IRC secundaria a hipertensión o diabetes (Eduards y Prozialeck 2009, Kim et al. 2015). Nuestros resultados no mostraron que el cadmio tenga un efecto significativo, pero sí podrían indicar que potencie el efecto del arsénico por sinergia.

De acuerdo con un estudio de la OMS (WHO 2016), la zona metropolitana del Valle de Toluca es hoy la segunda zona urbana más contaminada de México. Estudios recientes informan la presencia de metales pesados como cadmio y plomo en el aire (Zarazúa-Ortega et al. 2013a, Macedo-Miranda et al. 2016). El curso del río Lerma-Santiago es uno de los más contaminados del país a consecuencia de las numerosas descargas industriales y municipales, así como los escurrimientos agrícolas, y en él se han encontrado metales como cromo, manganeso, hierro, cobre, zinc y plomo (ZarazúaOrtega 2013b).

El arsénico ha sido poco estudiado en el Estado de México, pero su ingestión es generalmente a través del agua potable. En este estudio se encontró que el $14 \%$ de la muestra (11 pacientes y tres testigos) tenían valores de arsénico en cabello por encima del límite aceptado, y la exposición a este elemento resultó un factor de riesgo significativo para el desarrollo de una IRCd anómala. Se requiere un estudio más amplio para identificar de manera particular las fuentes de exposición a arsénico. El análisis de la figura 1 sugiere que estas fuentes son de origen ambiental (asociadas a la cuenca del Lerma) e industrial, ya que en el municipio Toluca (donde hay varios corredores industriales) se observa una alta concentración de casos. Sin embargo, se requieren más estudios para corroborar lo anterior.

\section{CONCLUSIONES}

Hay una asociación estadísticamente significativa entre la IRCd presente en el poniente del Estado de México y el consumo de tabaco, la exposición ocupacional en construcción y herrería, y la exposición a arsénico. Los resultados indican además que la exposición ocupacional de los trabajadores en la industria del papel, el consumo de paracetamol y naproxeno, así como la exposición a cadmio, podrían ser también factores de importancia; no obstante, para corroborarlo se requiere ampliar el tamaño de la muestra. Aunque las condiciones geográficas y climatológicas del estado de México son diferentes a las de aquellos sitios en Centroamérica donde se observa MeN, la IRCd presente en este estado es bastante similar, por lo que se requieren estudios a mayor escala.

\section{AGRADECIMIENTOS}

Los autores agradecen la asistencia financiera del Fondo de Fomento y Desarrollo de la Investigación Científica y Tecnológica de la Universidad Autónoma del Estado de México a través del proyecto de investigación "Metales pesados e insuficiencia renal crónica. Estudio en adultos jóvenes del Estado de México", con clave 3896/2015E, así como el apoyo de la Red Académica y de Colaboración "Microbiología y química en ciencias de la salud", con clave 1039/2014RIFC". 


\section{REFERENCIAS}

Aburto-Medina A., Castillo D., Ortiz I., Hernández E., List R. y Adetutu E. (2015). Microbial community and pollutants survey in sediments of biologically important wetlands in Lerma, Mexico. Rev. Int. Contam. Ambie. 31(1), 7-22.

Barceló-Quintal I., López-Galván E., Solís-Correa H., Domínguez-Mariani E. y Gómez-Salazar S. (2012). Water quality assessment of José Antonio Alzate Dam, the Lerma River and its tributaries in the State of Mexico, Mexico. J. Environ. Prot. 3 (8), 878-888. DOI: $10.4236 /$ jep.2012.328103

Brito E.M., De la Cruz Barrón M., Caretta C.A., GoñiUrriza M., Andrade L.H., Cuevas-Rodríguez G., Malm O., Torres J.P., Simon M. y Guyoneaud R. (2015). Impact of hydrocarbons, PCBs and heavy metals on bacterial communities in Lerma River, Salamanca, Mexico: Investigation of hydrocarbon degradation potential. Sci. Total Environ. 521-522, 1-10.

DOI: 10.1016/j.scitotenv.2015.02.098

Brooks D.R., Ramírez-Rubio O. y Amador J.J. (2012). CKD in Central America: A hot issue. Am. J. Kidney Dis. 59 (4) 481-484.

DOI: 10.1053/j.ajkd.2012.01.005

Carvalho A.S.C., Santos A.S., Pereira S.F.P. y Alves C.N. (2009). Levels of $\mathrm{As}, \mathrm{Cd}, \mathrm{Pb}$ and $\mathrm{Hg}$ found in the hair from people living in Altamira, Pará, Brazil: Environmental implications in the Belo Monte Area. J. Braz. Chem. Soc. 20 (6), 1153-1163.

DOI: $10.1590 / \mathrm{S} 0103-50532009000600022$

Edwards J.R., y Prozialeck W.C. (2009) Cadmium, diabetes and chronic kidney disease. Toxicol. Appl. Pharmacol. 238 (3), 289-293.

DOI: $10.1016 /$ j.taap.2009.03.007

Elinder C.-G., Wernerson A. y Wijkström J. (2015). Mesoamerican nephropathy (MeN): A 'new' chronic kidney disease related to occupational heat exposure with repeated deprivation of salts and water. Int. J. Nephrol. Kidney Failure 1(2).

DOI: $10.16966 / 2380-5498.109$

Jayasumana M.A.C.S., Paranagama P. A., Amarasinghe M.D., Wijewardane K.M.R.C., Dahanayake K.S., Fonseka S.I., Rajakaruna K.D.L.M.P., Mahamithawa A.M.P., Samarasinghe U.D. y Senanayake V.K. (2013). Possible link of chronic arsenic toxicity with chronic kidney disease of unknown etiology in Sri Lanka. Journal of Natural Sciences Research 3, 64-73.

Jayatilake N., Mendis S, Maheepala P. y Mehta F.R. (2013). Chronic kidney disease of uncertain aetiology: prevalence and causative factors in a developing country. BMC Nephrology 14, 180.

DOI: $10.1186 / 1471-2369-14-180$
Kim N.H., Hyun Y.Y., Lee K.B., Chang Y., Rhu S., Oh K.H. y Ahn C. (2015). Environmental heavy metal exposure and chronic kidney disease in the general population. J. Korean Med. Sci. 30 (3), 272-277.

DOI: $10.3346 / \mathrm{jkms} .215 .30 .3 .272$

Macedo-Miranda G., Ávila-Pérez P., Gil-Vargas P., Zarazúa G., Sánchez-Meza J. C., Zepeda-Gómez C. y Tejeda S. (2016) Accumulation of heavy metals in mosses: A biomonitoring study. Springerplus 5, 715. DOI: $10.1186 / \mathrm{s} 40064-016-2524-7$

Méndez-Durán A., Méndez-Bueno J.F., Tapia-Yáñez T., Muñoz-Montes A. y Aguilar-Sánchez L. (2010). Epidemiología de la insuficiencia renal crónica en México (Epidemiology of chronic kidney failure in Mexico). Dial. Traspl. 31 (1), 7-11.

DOI: $10.1016 / \mathrm{S} 1886-2845(10) 70004-7$

Noble A., Amerasinghe P., Manthrithilake H. y Arasalingam S. (2014). Review of literature on chronic kidney disease of unknown etiology $(\mathrm{CKDu})$ in Sri Lanka. IWMI Working Paper 158. International Water Management Institute, Colombo, Sri Lanka, 41 pp. DOI: $10.5337 / 2014.206$

Plantinga L., Grubbs V., Sarkar U., Hsu C.Y., Hedgeman E., Robinson B., Saran R., Geiss L., Ríos-Burrows N., Eberhardt M. y Powe N. (2011). Nonsteroidal antiinflammatory drug use among persons with chronic kidney disease in the United States. Ann. Fam. Med. 9 (5), 423-430.

DOI: $10.1370 / \mathrm{afm} .1302$

Redmon J.H., Elledge M.F., Womack D.S., Wickremashinghe R., Wanigasuriya K.P., Peiris-John R.J., Lunyera J., Smith K., Raymer J.H. y Levine K.E. (2014). Additional perspectives on chronic kidney disease of unknown aetiology (CKDu) in Sri Lanka - Lessons learned from the WHO CKDu population prevalence study. BMC Nephrology 15, 125.

DOI: $10.1186 / 1471-2369-15-125$

Tejeda S., Zarazúa-Ortega G., Ávila-Pérez P., García-Mejía A., Carapia-Morales L. y Díaz-Delgado C. (2006). Major and trace elements in sediments of the upper course of Lerma river. J. Radioanal. Nucl. Chem. 270 (1), 9-14. DOI: 10.1007/s10967-006-0342-Z

Trasande L., Cortes J.E., Landrigan P.J., Abercrombie M.I., Bopp R.F. y Cifuentes E. (2010). Methylmercury exposure in a subsistence fishing community in Lake Chapala, Mexico: An ecological approach. Environ. Health 9,1 .

DOI: 10.1186/1476-069X-9-1

Wesseling C., Crowe J., Hogstedt C., Jakobsson K., Lucas R. y Wegman D. (2013). Mesoamerican nephropaty. Report from the First International Research Workshop on MeN. Central American Institute for Studies on Toxic Substances (IRET-UNA), Costa Rica, 254 pp. 
WHO (2016). Global urban ambient air pollution database (update 2016). World Health Organization [en línea]. http://www.who.int/entity/phe/health_topics/ outdoorair/databases/who-aap-database-may2016. xlsx?ua $=1$ 14/06/2017

Zarazúa-Ortega G., Poblano-Bata J., Tejeda-Vega S., Ávila-Pérez P., Zepeda-Gómez C., Ortiz-Oliveros H. y Macedo-Miranda G. (2013a). Assessment of spatial variability of heavy metals in metropolitan zone of
Toluca Valley, Mexico, using the biomonitoring technique in mosses and TXRF analysis. Sci. World J. Article ID 426492. DOI: 10.1155/2013/426492

Zarazúa-Ortega G., Ávila-Pérez P., Tejeda S., ValdiviaBarrientos M., Zepeda-Gómez C. y Macedo-Miranda G. (2013b). Evaluación de los metales pesados Cr, Mn, $\mathrm{Fe}, \mathrm{Cu}, \mathrm{Zn}$ y $\mathrm{Pb}$ en sombrerillo de agua (Hydrocotyle ranunculoides) del curso alto del Río Lerma, México. Rev. Int. Contam. Ambie. 29 (Sup. 2), 17-24. 